\title{
Changement organisationnel et reconception de l'organisation : des ressources aux capabilités
}

Organizational change and organizational redesign-in-use: from resources to capabilities

Justine Arnoud et Pierre Falzon

\section{CpenEdition}

Journals

Édition électronique

URL : http://journals.openedition.org/activites/760

DOI : $10.4000 /$ activites.760

ISSN : 1765-2723

Éditeur

ARPACT - Association Recherches et Pratiques sur les ACTivités

Référence électronique

Justine Arnoud et Pierre Falzon, « Changement organisationnel et reconception de l'organisation : des ressources aux capabilités », Activités [En ligne], 10-2 | Octobre 2013, mis en ligne le 15 octobre 2013, consulté le 19 avril 2019. URL : http://journals.openedition.org/activites/760 ; DOI : 10.4000/ activites.760

\section{(c) $($ ) $(9)$}

Activités est mis à disposition selon les termes de la licence Creative Commons Attribution - Pas d'Utilisation Commerciale - Pas de Modification 4.0 International. 


\title{
ChaEgemeEt orgaEisatioEFel et recoEceptioE de l'orgaEisatioE : des ressources aux capabilités
}

\author{
JustiEe ArEoud \\ Conservatoire National des Arts et Métiers (CNAM), CRTD, 75005 Paris \\ Auteur correspondant justine.arnoud@yahoo.fr \\ Pierre FalzoE \\ Conservatoire National des Arts et Métiers (CNAM), CRTD, 75005 Paris
}

\begin{abstract}
OrgaEizatioEal chaFge aEd orgaEizatioEal redesigE-iE-use: from resources to capabilities. This paper aims to describe the processes of organizational redesign-in-use as they are carried out by human operators following an organizational change. The purpose of these processes is to ensure production quality and to protect instances of cooperation which the structure, as it was designed, tends to ignore. The qualitative methodology we applied here allowed us to identify certain characteristics of the structure and to highlight the role of redesign processes. Our main results show that operators attempt to convert the organization's resources into "capabilities", i.e. into effective possibilities to "do the job" and "do the job well". However, the rigid nature of the organization hinders such attempts, which are not always known or discussed within the organization. One goal of ergonomic intervention is therefore to provide evidence of these attempts and the means to discuss them, in order to implement a methodology which will support organizational redesign-in-use and to place human actors at the heart of organizational change.
\end{abstract}

KEYWORDS

organization, organizational change, redesign-in-use, capabilities, ergonomic intervention

\section{1.- IEtroductioE}

Cette intervention s'insère dans le cadre d'un changement organisationnel qui s'est traduit par le regroupement des fonctions paie et comptabilité d'un grand groupe au sein d'un Centre de Services Partagés (CSP). Un CSP est une entité juridiquement autonome qui réalise une ou plusieurs fonctions dites «support» du groupe auquel cette entité appartient (de La Villarmois \& Tondeur, 2002). Cette forme organisationnelle a connu un succès important dans les entreprises cette dernière décennie (Schulz, Hochstein, Uebernickel, \& Brenner, 2009) à tel point qu'un «modèle CSP »s'est progressivement constitué et a été largement reproduit. Dans l'entreprise où nous avons réalisé l'étude, le changement a consisté à appliquer de façon mimétique (DiMaggio \& Powell, 1983) ce modèle supposé avoir fait ses preuves chez les concurrents. Ce «modèle » est assimilable à ce que Boussard (2013) appelle un « dispositif de gestion » proposé par des consultants aux professionnels de l'organisation et qui contient tout à la fois la définition du problème, son diagnostic et sa solution. Le changement a alors été le fruit de la seule volonté de dirigeants persuadés de l'efficacité d'un tel modèle et a été imposé aux acteurs.

Nombre d'ouvrages de management véhiculent encore l'idée d'un changement «comme étant le fait de décideurs installés au sommet de l'entreprise qui, forts d'une vision globale, ont pour tâche d'en mettre en œuvre les conséquences organisationnelles» (Sardas \& 
Guénette, 2005, p. 11). Ainsi, le changement est-il considéré comme le passage d'un état A stable à un état B connu et pré-formalisé (Bernoux, 2004) et sa mise en œuvre peut alors être effectuée par des cabinets de conseil qui attribuent toutes les vertus à l'état B (Dugué, 2006). Dans ce cas, de nouvelles règles sont prescrites. La constitution de ces règles s'appuie rarement sur l'analyse des régulations qui ont eu lieu à l'état $\mathrm{A}$ et qui ont pu conduire à la production de « règles effectives », acceptables par tous (Reynaud, 1995). Pour autant, quel que soit le formalisme imposé par la nouvelle structure, de nombreuses études (Bernoux, 2004 ; Cuvelier, 2007 ; Gilbert, 2008 ; Sardas \& Lefebvre, 2005) font état de l'importance de l'informel, des interactions et des mécanismes d'appropriation dans l'émergence de nouveaux fonctionnements organisationnels. Autrement dit, il y aura toujours un écart entre les usages prévus en conception et l'usage et l'appropriation de la nouvelle organisation en situation.

À la lumière des travaux sur la reconception organisationnelle dans l'usage (Rabardel, 1995) et des travaux récents sur les environnements capacitants (Falzon, 2005 ; 2013 ; FernaguOudet, 2012), nous faisons l'hypothèse que les acteurs vont progressivement remettre l'organisation «à leur main» pour l'adapter aux situations variées rencontrées (Petit \& Coutarel, 2013). Cette «mise en main» est fonction des ressources individuelles et organisationnelles disponibles, mais également de la présence de «facteurs de conversion » (Fernagu-Oudet, 2012) autorisant la conversion des ressources en réalisations concrètes. Les «facteurs de conversion » désignent les dispositifs humains, organisationnels, techniques et sociaux qui vont permettre la transformation des ressources et moyens disponibles en facultés personnelles de déployer un travail pour lequel chacun accorde de la valeur. En l'absence de ces facteurs, la «mise en main » de l'organisation par les opérateurs s'avérera coûteuse. Le concept de «facteur de conversion» ainsi que le lien entre «facteur de conversion» et «qualité du travail» seront précisés dans un cadre théorique plus large autour de l'organisation et de sa conception.

À partir d'une analyse ergonomique menée dans le cadre de la mise en place d'un CSP, l'objectif sera d'identifier et mettre à jour les ressources de la nouvelle organisation CSP et les facteurs qui facilitent ou entravent la conversion de ces ressources en « capabilités ». Sur la base de ce diagnostic, nous discuterons les possibilités d'intervention visant la «mise en route » de facteurs de conversion positifs et durables. Dans ce cadre, le changement est assimilé à un processus de conception (Guibert, 2009; Sardas \& Lefebvre, 2005) conception qui se poursuit dans l'usage et qui permet la construction progressive de la nouvelle organisation.

Plus généralement, il s'agit de replacer les acteurs au cœur du changement et de promouvoir la conception d'organisations «adaptables » - la conception se poursuit dans l'usage - et « capacitantes »- des ressources sont disponibles et effectives.

\section{2.- OrgaEisatioE et coEceptioE orgaEisatioEFelle}

\section{1.- OrgaEisatioE et chaEgemeEt orgaEisatioEFel}

À un niveau macro-social, la théorie de la structuration (Giddens, 1987) pose une relation dialectique entre les acteurs et les structures sociales, les propriétés structurelles des systèmes sociaux étant à la fois «le médium et le résultat des pratiques qu'elles organisent » (Ibid., p. 75). Cette théorie constitue un tournant dans la recherche sur les organisations ${ }^{1}$, conduisant à une modélisation nouvelle des phénomènes organisationnels et des systèmes de pilotage associés. La structure organisationnelle perd son caractère purement déterministe et

1 Quelles que soient les formes juridiques et les frontières formelles des institutions concernées (hôpitaux, écoles publics, entreprises privées etc.), l' « intervention conjointe d'acteurs multiples » constitue de fait une organisation (Lorino, 2009). 
un intérêt croissant est porté aux pratiques des acteurs et à leurs interactions.

En sciences de gestion et en ergonomie, l'organisation est alors modélisée par «une structure et des interactions sociales qui agissent avec et sur la structure » (Petit, 2005, p. 172). Dans cette perspective, l'organisation ou les systèmes de gestion s'apparentent à des instruments (Rabardel, 1995) combinant des artefacts organisationnels qui composent la structure (les règles formelles et dispositifs qui guident les acteurs) et des schèmes d'utilisation (les modes d'usage de ces artefacts) susceptibles de faire évoluer la structure (Lorino, 2005 ; Petit, 2005).

Selon ces acceptions, «piloter» une organisation revient à «aller» dans l'action et la capitaliser dans la structure (Autissier \& Wacheux, 2000). De nouvelles questions émergent avec une acuité particulière lors des périodes de changement: la structure se construit-elle dans le mouvement de l'action ? Faut-il laisser émerger de nouveaux fonctionnements avant de modifier la structure ou basculer rapidement dans une nouvelle organisation? (Ibid.; Sardas \& Lefebvre, 2005). «Laisser émerger de nouveaux fonctionnements » revient à considérer l'organisation non comme une structure figée, mais comme un «instrument» (Rabardel, 1995) «malléable » par l'opérateur-utilisateur (Petit \& Dugué, 2013).

Pour autant, le changement organisationnel ${ }^{2}$ est loin d'être appréhendé de la sorte. Dans la pratique, les changements restent accrochés à un modèle "mécaniste » de l'organisation (Petit, 2005) où la seule modification de la structure et son acceptation par les acteurs suffisent pour assurer son efficacité.

\section{2.- L'Homme chaFge l'orgaEisatioE}

Il apparaît pourtant illusoire de penser que «la» bonne organisation est prévisible à l'avance : "même dans les situations les plus contraignantes, les individus se créent des marges d'autonomie. C'est dans ces marges, articulées sur les contraintes de l'organisation, mais s'en démarquant en les modifiant, que se constitue le changement » (Bernoux, 2004, p. 11). Autrement dit, tout système, dans la pratique, dérive des choix initiaux des concepteurs (Wilson, 2012). L'ergonomie défend depuis longtemps une vision de l'opérateur comme créateur de son propre travail (Falzon, 1996) et, in fine, de sa propre organisation qu'il va chercher à «mettre à sa main » et à adapter aux situations variées qu'il peut rencontrer (Coutarel \& Petit, 2009). La nouvelle organisation, aussi formalisée soit-elle, sera confrontée à des tentatives d'adaptation et de reconception. Toute décision de changement subira des modifications (Bernoux, 2004). Dans les faits, cela se traduit concrètement par différentes pratiques :

— Les activités méta-fonctionnelles. Au cours du travail, les opérateurs, confrontés à des difficultés fonctionnelles lors de l'exécution des tâches, vont construire spontanément de nouveaux outils et de nouveaux savoirs (Falzon, Sauvagnac, \& Chatigny, 1996) « destinés à une utilisation ultérieure éventuelle et visant à faciliter l'exécution de la tâche ou à améliorer la performance » (Falzon, 1994, p. 2). Ces activités sont déclenchées par des dysfonctionnements (problèmes nouveaux, outils inadaptés, manque d'information...) et sont susceptibles de contribuer à la transformation de l'organisation (Falzon, 1996) ;

— Les migrations de pratiques. Habituellement utilisé pour expliquer la survenue d'accidents, le modèle des migrations de pratiques développé par Rasmussen (1997) explique plus généralement les pressions (environnementales, commerciales, recherches de bénéfices individuels...) présentes au sein des organisations à l'origine de modifications de comportements, de migration de pratiques. Ces migrations apparaissent

2 Par « changement organisationnel » nous entendons « les projets qui passent concrètement pour les acteurs par une évolution de leurs contenus d'activité et de leurs modes d'interaction » (Sardas \& Lefebvre, 2005, p. 256). 
nécessaires pour s'adapter aux tensions antagonistes et aux contraintes de travail. Dans ce modèle, l'activité de l'opérateur «prend la forme d'une autonomie aux contours incertains, migrant vers des limites du fait de tensions » (Valot, 2001, p. 26) ;

- L'invention de nouveaux usages. Les utilisateurs des nouveaux dispositifs organisationnels et techniques inventeront de nouveaux usages (Rabardel, 1995). L'organisation pouvant être considérée comme un instrument, ce dernier peut être élaboré, institué, transformé par le sujet y compris lorsque celui-ci est fondé sur un artefact (ici la structure organisationnelle) hyper-normé (Ibid.). Le développement de l'organisation se concrétise dans un processus de genèse entre la structure et les actions des acteurs (Petit, 2005). Ces «genèses » organisationnelles sont dirigées vers les sujets eux-mêmes par le développement de nouveaux schèmes (dimension instrumentation) et vers l'artefact par sa transformation (dimension instrumentalisation) (Rabardel, 1995). Elles traduisent donc en partie l'idée que l'organisation est progressivement retravaillée par ses acteurs (de Geuser, communication personnelle) ;

- La réélaboration des règles. Plusieurs types de règles coexistent au sein d'une organisation: des règles de contrôle, explicites et officielles et des règles autonomes, implicites (Reynaud, 1988). Ces dernières sont élaborées par les travailleurs qui assurent le fonctionnement quotidien de l'organisation. Le changement est à l'origine de la création de nouvelles règles : "c'est dans l'acceptation d'une confrontation entre ces deux régulations qu'émergeront de nouveaux fonctionnements » (Gilbert, 2008, p. 42). Cette régulation peut avoir lieu au cours même de l'activité (les régulations chaudes) ou au sein d'espaces dédiés (les régulations froides) (de Terssac \& Lompré, 1996).

L'ensemble de ces activités peut être défini comme un «travail d'organisation » visant à inventer des solutions singulières face à un problème particulier (de Terssac, 2003). La plupart du temps, ce travail est clandestin, parfois toléré (les migrations de pratiques et les régulations autonomes), mais rarement reconnu. Les pratiques décrites sont même souvent assimilées à des détournements, des fautes, des erreurs. Sans pour autant tomber dans l'extrême inverse visant à considérer l'ensemble de ces pratiques comme souhaitable et optimale, de nombreux auteurs montrent l'importance de considérer ces activités comme des tentatives d'amélioration qu'il convient d'identifier (Detchessahar, Clergeau, Devigne, Dumond, Honoré et al., 2006), de reconnaître et d'accompagner : "en France, l'intelligence des travailleurs est massivement utilisée à gérer dans l'ombre ce que l'organisation officielle ne prend pas en charge. On pourrait imaginer de l'utiliser au grand jour pour améliorer l'organisation » (Daniellou, 2006, p. 63). Comment ?

\section{3.- La coEceptioE d'orgaEisatioEs « adaptables » et « capacitaEtes »}

Il convient de placer les acteurs au centre des moyens pour guider les changements (Petit, 2005). Trois approches sont possibles (Falzon, 1996, 2005) : ces approches tiennent compte de l'activité des «utilisateurs» de l'organisation et traduisent également les différentes contributions, au cours du temps, des ergonomes à la conception :

— l'approche classique par «systèmes adaptés » où la conception intègre des usages anticipés a priori : il convient pour cela de prévoir l'activité, signaler des formes de variabilités probables dans le futur système et de définir ensuite «une» bonne organisation. L'idée est que «tout artefact cristallise une connaissance, une représentation, et au sens plus large un modèle de l'utilisateur et de son activité (...). Ces modèles peuvent être sources de difficultés pour les personnes s'ils sont faux ou insuffisants » (Béguin, 2008, pp. 373-374; 2013) et ne tiennent pas compte des usages qui se développeront;

— l'approche par «systèmes adaptables » où la conception «se poursuit dans l'usage » (Rabardel, 1995): il convient pour cela de prévoir des marges de manœuvre pour permettre le déploiement de l'activité (Coutarel, 2004 ; Daniellou, 1992). La bonne 
organisation devient celle que l'on peut mettre à sa main (Petit \& Coutarel, 2013). L'idée est alors de « concevoir des systèmes suffisamment plastiques pour laisser, à l'activité en situation, des degrés de liberté » (Béguin, 2008, p. 377) ;

— l'approche «développementale» où les usages se construisent au cours même de la conception à travers le dialogue et la confrontation d'acteurs multiples : cette approche « cherche à articuler dans un même mouvement, le développement des situations (artefact et/ou organisation) par les concepteurs et le développement des ressources de leur action par les opérateurs » (Béguin \& Cerf, 2004, p. 61). L'enjeu « consiste à concevoir non une nouvelle organisation, mais un dispositif d'expérimentation et d'apprentissage, pour susciter et favoriser de nouveaux fonctionnements organisationnels » (Sardas \& Lefebvre, 2005, p. 285).

Cette dernière approche, très séduisante, apparaît toutefois difficilement applicable aux changements tels qu'ils sont menés dans les organisations aujourd'hui : atteinte rapide d'une cible $\mathrm{B}$ prédéfinie, modèle de prise de décision managérial... Et, bien souvent, les ergonomes sont appelés «après-coup » sans être associés au processus de conception (Cuvelier, 2007). L'objectif est alors d'enrichir l'approche «systèmes adaptables » par les travaux récents autour du modèle des « capabilités » (Sen, 2009).

Les «capabilités» sont définies comme les fonctionnements humains disponibles à un individu, qu'il en fasse usage ou non (Falzon, 2005). Concevoir « un espace au sein duquel l'activité pourra se déployer» (Daniellou, 1992) revient à laisser des marges de manœuvre en termes de ressources, mais également en termes de «facteurs de conversion» de ces ressources en «capabilités » (Fernagu-Oudet, 2012). Les facteurs de conversion renvoient à « l'ensemble des facteurs qui facilitent ou entravent la capacité d'un individu à faire usage des ressources à sa disposition pour les convertir en réalisations concrètes » (Ibid., p. 10). L'enjeu est alors de concevoir des systèmes « capacitants » qui facilitent l'accroissement de capabilités. Concrètement, cet accroissement n'est possible qu'à condition que l'organisation mette à disposition des ressources, mais également prévoit des «facteurs de conversion » afin de transformer les potentialités - individuelles, collectives, technico-organisationnelles - en possibilités effectives. Autrement dit, elle doit proposer des «environnements capacitants » (Falzon, 2005 ; 2013) afin de fournir aux individus l'occasion de développer de nouveaux savoir-faire et de nouvelles compétences, d'élargir leurs possibilités d'action, leur degré de contrôle sur leur tâche (Pavageau, Nascimento, \& Falzon, 2007). Illustrons notre propos à travers la figure 1 suivante :

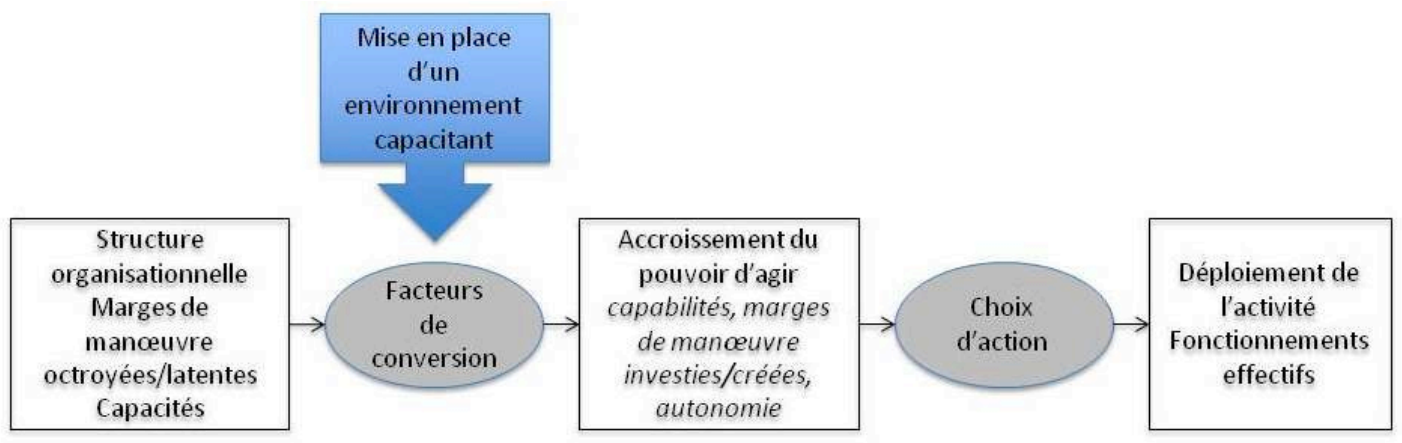

Figure 1: L'approche par les capabilités appliquée à l'organisation (adapté de Bonvin \& Farvaque, 2007 et Fernagu-Oudet, 2012)

Figure 1: The capability approach applied to organization (adapted from Bonvin \& Farvaque, 2007 and Fernagu-Oudet, 2012) 
Au sein de l'organisation, la seule existence de ressources :

— internes au sujet : les capacités (savoirs et savoir-faire), les caractéristiques individuelles (expérience, niveau de formation, etc.) ;

- et externes au sujet : la structure organisationnelle - règles et dispositifs au sens large -, les collègues et les marges de manœuvre prévues par la structure ou latentes, ne suffit pas. Celles-ci doivent être converties en «capabilités »- pouvoir effectif de faire quelque chose - et cette conversion s'opère à travers la mise en place d'un environnement capacitant qui permet aux individus et aux collectifs de développer leurs savoirs, leur santé, de réussir et de progresser. Ce faisant, l'autonomie s'accroît et les marges de manœuvre deviennent réellement investies et/ou créées. Ceci élargit le rayon d'actions et de choix effectifs, autrement dit le pouvoir d'agir qui va autoriser le déploiement de l'activité.

La mise en place de facteurs de conversion positifs permet ainsi à l'individu d'accomplir des fonctionnements pour lesquels il attribue de la valeur, c'est-à-dire un travail de qualité. La «mise en main » de l'organisation par l'opérateur peut se traduire comme une tentative de celui-ci ou d'un collectif de convertir les ressources en capabilités, mais, sans les conditions favorables d'un environnement adaptable et capacitant, cette conversion peut se révéler difficile et coûteuse.

\section{3.- Le terraiE de recherche}

Les résultats que nous proposons dans cet article sont issus d'une intervention ergonomique menée dans un Groupe spécialisé dans les services liés à l'environnement. Ce groupe est structuré autour de quatre principales activités qui façonnent une organisation autour de quatre «divisions » : l'eau, la propreté, l'énergie et les transports. Chacune de ces divisions est composée de multiples établissements et filiales répartis sur le territoire français et à l'international. Plus de 100000 collaborateurs travaillent dans ce groupe en France, plus de 200000 à l'étranger, majoritairement des ouvriers et agents d'exploitation (62\% des effectifs globaux).

\section{1.- Le coEtexte de l'iEterveEtioE}

Depuis quelques années, un vaste plan de transformation a été lancé par le président du groupe. Ce plan a pour objectif de répondre aux impératifs de la crise financière et à la nécessaire réduction de l'endettement. Différentes mesures ont été adoptées : parmi elles, le siège a décidé de regrouper les fonctions comptabilité fournisseur/générale et paie au sein d'un Centre de Services Partagés (CSP). Cette décision s'insère dans un objectif global de regroupement de fonctions dites «support» (marketing, formation, recherche-innovation, etc.) au sein d'entités juridiquement distinctes visant à favoriser les synergies entre les quatre divisions et à donner de la cohérence au groupe.

Un CSP est une entité juridiquement autonome qui réalise une ou plusieurs fonctions dites support de l'organisation à laquelle cette entité appartient (de La Villarmois \& Tondeur, 2002). Le projet «CSP » a débuté en 2007 et poursuit plusieurs objectifs : la standardisation et l'uniformisation des processus paie et comptabilité permettant l'industrialisation de ces fonctions et le rapprochement des pratiques des divisions, la réalisation d'économies d'échelle induites par la mutualisation, la possibilité pour les établissements et filiales (désignés par la suite sous le terme «local») de se concentrer sur leur cœur de métier. Un contrat de service a été signé entre le CSP et le local où les comptables et gestionnaires du CSP deviennent des prestataires de service et les prestations sont facturées aux bénéficiaires, les «clients» en local. Au total quatre CSP ont été ouverts, un CSP pilote à Lyon puis trois autres à Nantes, Rueil et Lille.

À notre arrivée, en 2010, la nouvelle organisation, telle que présentée sur la figure 2, connaissait de grandes difficultés. Les objectifs n'étaient pas atteints, les opérateurs 
(gestionnaires et correspondants) exprimaient des difficultés à travailler dans le nouveau contexte et les «clients » étaient mécontents.

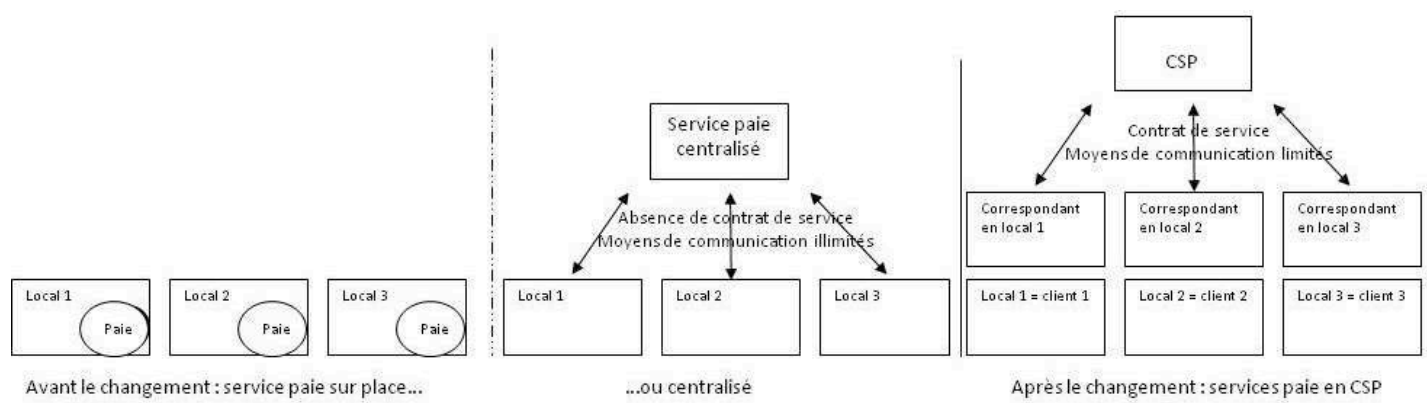

Figure $2:$ L'organisation avant / après le changement. Le cas de la fonction paie

Figure 2: The organization before / after the change. The case of the pay function

La direction des ressources humaines du CSP et les directions en local souhaitaient comprendre les raisons de ces difficultés et améliorer le fonctionnement de la nouvelle organisation. C'est dans ce contexte que nous avons reçu une demande conjointe de ces deux directions qui souhaitaient être accompagnées dans cette réflexion. L'intérêt de cette demande était de pouvoir intervenir lors d'un changement organisationnel en cours puisque le transfert des activités paie/comptabilité n'était pas terminé en 2010. Des situations «avant changement » étaient donc toujours observables.

\section{2.- Le groupe d'opérateurs étudiés et leurs tâches}

À la demande du local, nous avons choisi de nous intéresser plus spécifiquement à l'activité paie qui semblait regrouper un grand nombre de difficultés. Trois services paie ont été sélectionnés. Ce choix a été réalisé en concertation avec les demandeurs de manière à appréhender au mieux le changement et ses conséquences. Un service a pu être observé avant son passage en CSP (service A). Deux autres services ont été choisis : le service paie du CSP «pilote» de Lyon où l'application du modèle CSP et des outils associés est la plus aboutie (service C) et le service paie du CSP de Rueil où les outils-cibles ne sont pas encore mis en place (service B). L'analyse préalable du fonctionnement des autres CSP a montré que le CSP de Nantes présentait des caractéristiques similaires à celui de Rueil et que le CSP de Lille n'avait pas encore en charge le traitement de la paie.

Au sein de ces 3 services, les gestionnaires paie ont en charge la production de la paie pour le compte d'établissements ou de filiales géographiquement éloignés :

— le service A : il s'agit d'un service paie "centralisé » au sein de la direction de la division Propreté dont le passage en CSP a été repoussé en 2014. Ce service a été choisi, car il présente les mêmes caractéristiques que les services $B$ et $C$ avant leur passage en CSP. En effet, dans la majorité des cas, les gestionnaires paie étaient préalablement rattachés à des services centralisés dans des sièges ou des «pôles». Seuls certains services étaient complètement décentralisés et dans ces rares cas, les gestionnaires étaient « sur place » (voir Figure 1). Ce service gère environ 5000 paies réparties entre 12 gestionnaires (environ 400 paies par personne) encadrés par une responsable d'équipe. Les gestionnaires réalisent la paie pour le compte d'établissements en local, mais ne sont pas des prestataires. Aucun contrat de service n'est signé et les gestionnaires peuvent entrer librement en contact avec leurs correspondants ou directement avec les salariés si nécessaire ;

— le service B : il s'agit du service paie du CSP de Rueil en charge des paies de la division 
Transport d'Ile-de-France. Le transfert des paies de cette division s'est étalé d'avril 2010 à juillet 2011. Aujourd'hui, 21 collaborateurs travaillent dans ce service dont 13 gestionnaires paies, quatre gestionnaires administratifs, trois responsables d'équipe, une responsable d'activité. L'équipe gère 4500 paies (environ 280 paies par gestionnaire). Les gestionnaires sont devenues des prestataires de service et réalisent la paie pour le compte de «clients ». Un contrat de service a été signé peu de temps après l'arrivée des équipes au sein du CSP. Les gestionnaires peuvent entrer en contact uniquement avec un correspondant désigné dans chaque établissement, de préférence via la messagerie électronique - les appels téléphoniques étant considérés comme une « perte de temps »;

— le service C : il s'agit du service paie du CSP «pilote » en charge des paies de l'ensemble de la division Propreté (censé accueillir en 2014 le service A). Le transfert des paies de cette division n'est donc pas encore terminé à l'heure actuelle. 9540 paies sont gérées par 28 gestionnaires (340 paies par gestionnaire en moyenne) répartis au sein de quatre équipes chacune encadrée par un responsable d'équipe. Une responsable d'activité dirige le service et a décidé de ne pas appliquer la séparation des tâches entres les gestionnaires paie / administratif initialement demandée par le siège. À la différence du service B, les équipes du service $\mathrm{C}$ travaillent sous le logiciel cible (HR Access) et échangent avec leurs correspondants à travers un outil spécifique $\left(\mathrm{Neocase}^{3}\right)$. Là encore, les correspondants sont devenus des «clients » et un contrat de service a été signé.

De manière générale, les gestionnaires paie ont en charge la production de la paie (le contrôle, le calcul, l'édition des bulletins, etc.). Lorsqu'une séparation des tâches a lieu (situation B), le gestionnaire administratif prend en charge une partie de l'activité des gestionnaires paie qui n'est pas directement liée à la production de la paie (création des fiches salariés, saisie de données relatives à la maladie, mise à jour des tableaux de bord, etc.).

Au-delà de ces trois services (A, B et C), nous avons souhaité élargir nos analyses à la population des « correspondants » au sein du local. Cette fonction a été spécifiquement créée lors de la mise en place des CSP. La plupart du temps, ce rôle a été confié aux assistants au sein du local et s'est traduit par l'ajout de nouvelles tâches en lien avec le CSP : transfert des documents, interlocuteur privilégié des salariés sur des questions relatives à la paie, etc. Les correspondants de la division transport en relation avec le service B ont été plus spécifiquement observés, sur la base du volontariat. De manière générale, le correspondant a en charge la tenue du planning, c'est-à-dire la collecte des temps et activités des salariés, et il doit transférer les documents utiles au CSP (feuilles d'arrêt maladie, d'accident du travail, justificatifs de transport, etc.).

\section{3.- Les motivatioEs et modalités de mise eE œuvre du chaEgemeEt}

Le changement a été motivé par des déterminants extérieurs : selon la direction, «le contexte économique tendu » a contraint le groupe à «trouver des moyens d'économiser, des moyens d'être plus performants ». Le siège a alors décidé de mettre en place un CSP, ce modèle « ayant fait ses preuves ». Ce changement «top-down » apparaît isomorphique (DiMaggio \& Powell, 1983) dans le sens où il est initié par la répétition de comportements qui, par le passé, ont été perçus par le marché comme performants : «on doit être les derniers du CAC40 à adopter cette démarche, on n'invente rien c'est un modèle qui a été prouvé » (Directeur CSP). La cible à atteindre - le CSP - est donc un modèle prédéfini avec des postulats : «pour être performants, les comptables doivent traiter 1000 factures par mois, les gestionnaires 500 paies par mois » (Directeur CSP). Le passage de A vers B a alors été remis

3 Neocase est une solution de services RH partagés permettant d'automatiser et d'orienter les demandes clients qui sont tracées en temps réel. A chaque demande est associé un temps de traitement que le prestataire de service s'engage à respecter. Cet outil permet également aux managers d'accroître leur visibilité sur les performances de leurs équipes via des rapports et tableaux de bord personnalisés. 
entre les mains d'un cabinet de conseil « spécialisé » dans la mise en place de ce modèle. La relation avec les Instances Représentatives du Personnel s'est traduite par une simple relation « information-consultation-avis » où l'avis recueilli (purement consultatif) a peu importé puisque le projet était élaboré, la cible fixée. Les opérateurs ont été tenus à l'écart du projet et il n'y a pas eu d'implication des usages dans la conception.

Le groupe a cherché à encourager les mobilités (à travers notamment l'ouverture de quatre CSP pour se rapprocher des salariés), mais les refus des opérateurs ont été nombreux en raison, d'une part, de l'éloignement géographique, et, d'autre part, de la représentation que les opérateurs avaient du travail au sein des CSP (crainte de la spécialisation et de l'appauvrissement des tâches). Certains établissements et filiales au sein du local ont interrogé la pertinence du changement et ont parfois freiné (voire refusé) le passage de leurs activités paie et comptabilité vers le CSP. Pour cette raison, le changement, initié en 2007, se poursuit encore aujourd'hui.

\section{4.- La méthode}

Pour répondre à la demande issue de la direction des ressources humaines des CSP et de la division transport d'Ile-de-France, nous avons effectué des analyses ergonomiques du travail des salariés dans les situations A, B, C et en local. L'objectif était d'évaluer la nouvelle organisation sous l'approche capabilités visant à :

— analyser la structure « CSP », ses règles et modalités de fonctionnement ;

— mettre à jour les ressources mises à la disposition des opérateurs au sein de la nouvelle organisation et les facteurs de conversion - positifs ou négatifs - des ressources en capabilités ;

— identifier les processus de reconception dans l'usage et la manière dont ces processus sont - ou non - discutés et réintégrés au sein de la structure.

\section{1.- Étape 1 : Caractériser la « structure » CSP}

Afin de comprendre le contexte général du changement, des entretiens ouverts ont été menés (voir Tableau 1) auprès de personnes impliquées dans la mise en place de la nouvelle organisation :

\begin{tabular}{|c|c|c|c|}
\hline Entité & Fonction & $\begin{array}{c}\text { Nombre d'entretiens } \\
\text { menés }\end{array}$ & Code \\
\hline $\begin{array}{l}\text { Siège du groupe ou sièges } \\
\text { des divisions }\end{array}$ & $\begin{array}{l}\text { - Directeur des relations sociales VE } \\
\text { - Directeur des relations sociales Eau } \\
\text { - Directeur prévention/santé/sécurité VE } \\
\text { - Responsable déploiement ERP Eau }\end{array}$ & 4 & $\mathrm{D} 1(\ldots)$ \\
\hline CSP & $\begin{array}{l}\text { - Directeur des quatre CSP } \\
\text { - Directeur de la performance } \\
\text { - Directeur des ressources humaines } \\
\text { - Directeur d'établissement } \\
\text { - Ancienne consultante ayant participé à la mise en } \\
\text { place du CSP }\end{array}$ & 7 & (...) à D9 \\
\hline \multirow[t]{2}{*}{$\begin{array}{l}\text { Local } \\
\text { Division Transport - Pôle } 78\end{array}$} & $\begin{array}{l}\text { - Responsable ressources humaines pôle } \\
\text { - Responsable administratif financier pôle } \\
\text { - Ancienne responsable d'équipe paie n'ayant pas } \\
\text { souhaité rejoindre le CSP }\end{array}$ & 4 & L1 à L3 \\
\hline & TOTAL & $\begin{array}{l}\mathbf{1 5} \text { (dont } 7 \text { enregistrés } \\
\text { et retranscrits et } 8 \\
\text { prises de notes) }\end{array}$ & $\begin{array}{l}\text { Durée : de } \\
1 \mathrm{~h} \text { à } 2 \mathrm{~h}\end{array}$ \\
\hline
\end{tabular}

Tableau 1 : Répartition des opérateurs rencontrés selon leur entité et leur fonction

Table 1: Distribution of the operators in terms of their unit and function

Au total 15 entretiens ont été menés et leur durée variait de $1 \mathrm{~h} 30$ à $2 \mathrm{~h}$ en fonction de la disponibilité des personnes. Les entretiens commençaient par la question : « comment s'est déroulé le passage en CSP?». L'interviewer laissait les personnes parler librement. 
L'objectif était de recueillir le point de vue de chacun sur le changement, ses enjeux, la manière de le mener et ses conséquences.

Au cours de ces entretiens, divers documents ont été collectés et notamment le contrat de service signé entre le service B et la division Transport. Ce contrat d'une soixantaine de pages a fait l'objet d'un traitement approfondi, car il a été rapidement perçu comme un support de lisibilité du changement. À travers ce document, quatre dimensions ont été analysées :

— le modèle de la relation entre le prestataire et le bénéficiaire : s'agit-il d'une relation de «client-fournisseur » ou de « partenariat»?;

— la performance : sur quelle approche de la performance le contrat est-il fondé ? Quels sont les indicateurs retenus?;

— les formes de la contractualisation: quel est le poids des éléments juridiques? Techniques ? Moraux ?;

— la prescription: quelles informations le contrat fournit-il sur la pré-organisation des tâches et activités? Quelle place le contrat accorde-t-il à la définition de marges de manœuvre?

\section{2.- Étape 2 : Évaluer les nouvelles situations de travail créées à travers une approche par les « ressources » et les « facteurs de conversion »}

Cette étape s'est déroulée de septembre 2010 à janvier 2012 et a combiné une pluralité de méthodes au sein des services $\mathrm{A}, \mathrm{B}$, et $\mathrm{C}$ précédemment décrits et des établissements de la division Transport. Des observations ouvertes ont eu lieu et ont permis la mise en place d'un guide d'entretiens et d'observations.

Un guide d'entretiens semi-directifs a été élaboré à partir de ces observations et du questionnaire proposé par Falzon, Nascimento et Pavageau (2008). Ce questionnaire propose trois indicateurs clés permettant de «détecter» un environnement capacitant autour des moyens favorables pour faire un travail de qualité, du sentiment d'utilité au travail et des possibilités d'apprentissage. Ces indicateurs sont complétés par des questions autour de la créativité (votre travail vous permet-il d'être créatif ?), l'efficacité (parvenez-vous à réaliser le travail demandé ?) et l'autonomie (pouvez-vous choisir la façon de procéder ?). Nous avons complété les entretiens avec les questions: «si vous deviez formuler des pistes d'amélioration de la nouvelle organisation, quelles seraient-elles?» et «selon vous, que signifie un travail de qualité ?». Ces entretiens ont été menés auprès de gestionnaires et responsables d'équipe au sein de la situation A (quatre personnes), B (six personnes) et C (sept personnes) et auprès de correspondants au sein du local (six personnes). Au total, 23 entretiens ont été menés, sur la base du volontariat. Leur durée variait de 40 minutes à $1 \mathrm{~h} 30$ en fonction du temps dont chacun disposait. Tous les entretiens ont été enregistrés et intégralement retranscrits pour faciliter leur traitement. Une analyse de contenu manuelle a été réalisée. Nous avons repris l'ensemble des protocoles et constitué une liste de 6 thèmes : profil des opérateurs, phase de transition, état des lieux de la situation actuelle, relation entre le local et le CSP, performance et critères d'évaluation, améliorations envisageables.

Une grille d'observations a également été élaborée où différents observables étaient systématiquement relevés :

— «les caractéristiques du travail» permettant de décrire les modalités d'exécution du traitement de la paie et les ressources mobilisées. Cet observable visait à analyser les libertés dans les façons de faire et à identifier les ressources mobilisées et mobilisables ;

— «le lien CSP-Local » visant à mieux appréhender cette relation en fonction des services analysés (avant/après CSP et au sein du service $\mathrm{C}$ «informatisé »). Cet observable a été choisi du fait de l'hypothèse que les difficultés rencontrées par la nouvelle organisation étaient en grande partie dues à la dégradation des relations entre le CSP et le local ; 
— les « régulations » effectuées par les acteurs pour mettre la nouvelle organisation « à leur main ». Cet observable répond au cadre théorique où le changement n'apparaît jamais subi par les acteurs. Ces derniers vont tenter de remettre l'organisation «à leur main». L'objectif est alors d'identifier ces tentatives et leur coût en fonction de leur caractère plus ou moins clandestin. Les résultats obtenus à partir de cet observable seront plus spécifiquement détaillés dans cet article. Le paragraphe suivant (étape 3) explicite le traitement de cet observable.

$\mathrm{Au}$ total, trois gestionnaires paie ont été observés dans la structure A (20 heures d'observation au total), quatre au sein de la structure B (27h) et quatre au sein de la structure C (20h).

\section{3.- Étape 3 : Identifier les effets des « régulations autonomes » sur la structure et la stratégie de la nouvelle organisation}

L'observable « régulations » sera plus spécifiquement analysé dans les résultats présentés ici. À travers cet observable, il s'agit de mettre à jour, par l'analyse ergonomique, les possibilités que trouvent ou s'octroient les salariés pour être capables de gérer leur quotidien. Concrètement, la grille d'observation a permis de relever, par la méthode papier crayon, l'ensemble de ce que nous avons appelé « régulations » et qui se traduisaient par des écarts à la prescription. Ces écarts étaient perceptibles grâce aux verbalisations conjointes à l'activité et grâce aux informations préalablement recueillies lors des entretiens et de l'étude du contrat de service. La prise de notes a ensuite été retranscrite sous Excel et deux grandes catégories ont été identifiées :

— la première catégorie "L'organisation CSP et ses procédures » a elle-même été divisée en 2 sous-catégories : «Remise en question de l'organisation du travail» et «Non application de procédures »;

— la seconde catégorie «La relation avec le local» a été divisée en 3 sous-catégories: «Non-respect des consignes de communication»; «Arrangements avec les correspondants »; « Nouvelles répartitions des portefeuilles ».

Nous avons ensuite repris les trois niveaux de déterminants de l'action proposés par Petit, Dugué et Daniellou (2011): niveau 1- Ce qui vient du cadre national (réglementation, politique du groupe, choix stratégiques); niveau 2- Ce qui relève de l'établissement local (structuration de l'entreprise, fonctionnement hiérarchique, gestion du personnel) ; niveau 3Ce qui concerne le travail au quotidien dans les services et les unités. À partir de cette distinction, nous avons cherché à identifier :

- la manière dont les régulations des acteurs (niveau 3) influençaient - ou non - les niveaux 2 et 1 ;

— et les modalités de confrontation des régulations de contrôle (niveaux 1 et 2) et des régulations autonomes (niveau 3 ).

\section{5.- Résultats}

Nous présenterons brièvement les principaux résultats obtenus à partir de l'étude du contrat de service et des caractéristiques de la nouvelle organisation (5.1.) puis nous détaillerons les processus de reconception dans l'usage au sein de ce contexte (5.2.).

\section{1.- La structure CSP et ses effets sur l'action des opérateurs}

\subsection{1.- Instauration d'une relation client-fournisseur et vision parcellaire du travail}

L'étude du contrat de service permet d'identifier l'existence d'une relation de type «clientfournisseur » (voir Tableau 2) caractérisée par : un contrat explicite, fermé, un besoin du client supposé entièrement défini et stable, un engagement du fournisseur à livrer un produit 
ou une prestation conforme au cahier des charges dans un délai donné et pour un coût donné (Cerf \& Falzon, 2005 ; Sardas, 2002) :

\begin{tabular}{|c|c|c|}
\hline \multicolumn{2}{|c|}{ Caractéristiques d'une relation client-fournisseur } & Extraits du contrat \\
\hline \multirow[t]{2}{*}{$\begin{array}{l}\text { Un contrat fermé et } \\
\text { complet }\end{array}$} & $\begin{array}{l}\text { Les clients doivent } \\
\text { précisément ce qu'ils veulent }\end{array}$ & \multirow[t]{2}{*}{$\begin{array}{l}\text { «Le CSP déclare, de manière expresse et } \\
\text { non équivoque, bien connaître les attentes et } \\
\text { les exigences du Client » }\end{array}$} \\
\hline & $\begin{array}{l}\text { Les fournisseurs doivent être certains } \\
\text { de pouvoir fournir le service désiré }\end{array}$ & \\
\hline $\begin{array}{l}\text { Pas de possibilité de } \\
\text { modifier les termes du } \\
\text { contrat pendant sa } \\
\text { réalisation }\end{array}$ & $\begin{array}{l}\text { Le contrat décrit de façon } \\
\text { «exhaustive et stable» ce que le } \\
\text { client attend / ce que le fournisseur } \\
\text { livre «sans qu'il soit besoin de } \\
\text { renégocier en cours d'exécution» }\end{array}$ & $\begin{array}{l}\text { Les engagements de part et d'autre sont } \\
\text { formalisés explicitement (cf. annexe } 4 \text { du } \\
\text { contrat « répartition des responsabilités »). } \\
\text { Seules de «nouvelles évolutions } \\
\text { fonctionnelles et organisationnelles des } \\
\text { entités» peuvent justifier la révision du } \\
\text { contrat sous forme d'«avenants» }\end{array}$ \\
\hline $\begin{array}{l}\text { Modifications } \mathrm{du} \\
\text { contrat } \\
\text { comme des erreurs }\end{array}$ & $\begin{array}{l}\text { Toute transformation est perçue } \\
\text { comme un « ratage » du contrat et } \\
\text { non comme un phénomène naturel } \\
\text { pouvant favoriser un apprentissage } \\
\text { des acteurs de la relation }\end{array}$ & $\begin{array}{l}\text { cf. la partie «pénalités » du contrat; ces } \\
\text { pénalités « représentent des sanctions } \\
\text { pécuniaires applicables en cas de } \\
\text { manquement à certaines obligations » }\end{array}$ \\
\hline $\begin{array}{l}\text { Tout incident est } \\
\text { perçu négativement }\end{array}$ & $\begin{array}{l}\text { Il n'y a pas d'objectif } \\
\text { d'apprentissage; les éventuels aléas } \\
\text { sont considérés comme des } \\
\text { perturbations qu'il faudra surmonter } \\
\text { au plus vite }\end{array}$ & $\begin{array}{l}\text { "Le client s'engage à résoudre avec } \\
\text { diligence tout problème ou difficulté en } \\
\text { relation avec ses engagements ou ses } \\
\text { responsabilités» }\end{array}$ \\
\hline
\end{tabular}

Tableau 2 : Le contrat de prestation de service : l'instauration d'une relation client-fournisseur

\section{Table 2: The service contract: a client-provider relationship}

Dans cette relation, le client sait précisément ce qu'il veut, le fournisseur a connaissance des souhaits du client et sait parfaitement comment y répondre. Il n'y a pas d'objectif d'apprentissage et les interactions sont limitées au seul transfert d'informations et de documents. Tout aléa est alors considéré comme un «manquement» au contrat qu'il faudra surmonter au plus vite sous peine de pénalités. La procéduralisation des relations qui en découle entraîne une formalisation claire des obligations de chacun et la recherche d'une évaluation «objective » et «quantifiable » de manière à encadrer au plus près le contenu de la prestation afin de mieux identifier les «manquements». La performance est fondée sur «la recherche permanente de productivité »: l'atteinte de cette performance permettrait la réduction des tarifs pratiqués - réduction pensée comme la seule variable de satisfaction du client. Par ailleurs, le contrat apparaît comme un véritable document prescripteur visant à « orienter l'action, à dire ce qui doit être fait dans des conditions données pour obtenir un certain résultat» (Leplat, 2004, p. 196). Les annexes concernant la répartition des responsabilités et des engagements permettent d'identifier une séparation des tâches à l'intérieur de la structure CSP (l'administration du personnel est séparée de la gestion de la paie) et une séparation nette des tâches entre les parties: chaque partie intervient sur un morceau du processus et est soumise à des contraintes de délais. La production de la paie est pensée comme pouvant se réaliser sans relation directe entre les co-contractants. Les entretiens menés avec la direction ont permis d'identifier que les moyens de communication entre les deux structures étaient limités aux seuls échanges par voie électronique pour «laisser des traces» et parce que les appels « ne sont pas nécessaires et font perdre du temps » (Direction CSP).

\section{1 .2.- Diminution des options disponibles}

L'analyse des entretiens et des observations montre que si des facteurs de conversion de ressources en capabilités sont identifiés au sein du service A (avant le passage en CSP), certains d'entre eux disparaissent et entravent l'usage des ressources dans les services B et/ou C. Prenons différents exemples. Au sein du service A, la gestion « de $\mathrm{A}$ à $\mathrm{Z}$ » de la paie est un élément fort de satisfaction : sur les quatre gestionnaires interviewés et observés, trois 
d'entre eux citent «la gestion de $\mathrm{A}$ à $\mathrm{Z}$ » comme premier critère d'appréciation générale du travail. Cette gestion globale confère aux gestionnaires un degré élevé de contrôle sur leur tâche et la possibilité de "réaliser des tâches différentes et variées ». Ce même critère est regretté $^{4}$ par cinq gestionnaires sur les six rencontrés au sein du service $\mathrm{B}$ où une séparation des tâches entre les gestionnaires administratif et paie est appliquée. Cette séparation fait perdre une partie de l' «histoire du salarié » et participe au sentiment d'être davantage dans un contexte administratif. Elle oblige chacun des acteurs à attendre le travail de l'autre et, lors des observations, cette attente apparaissait source de tensions. Au sein du local, la multiplicité des interlocuteurs et la question du «qui fait quoi » est une contrainte évoquée par cinq interlocuteurs sur les huit rencontrés - interlocuteurs en contact avec le service B.

Au sein du service A, cette gestion complète des dossiers s'accompagne d'une autonomie sur l'ensemble du processus. Si le travail est réalisé sous forte contrainte temporelle (bulletins de paie à sortir à une date précise chaque mois), les gestionnaires ont la possibilité de pouvoir s'organiser comme elles le souhaitent et de recueillir, comme elles l'entendent, les informations dont elles ont besoin auprès de différents interlocuteurs en local. Par ailleurs, les observations ont permis de révéler que chaque gestionnaire a développé une façon de travailler qui lui convient personnellement : vérification des paies à l'aide des bulletins du mois précédent, impression des bulletins en cours et comparaison avec les éléments envoyés par le local, etc.

Cette autonomie est réduite au sein de la nouvelle organisation.

D'après les gestionnaires interrogés au sein des services $\mathrm{B}$ et $\mathrm{C}$, les procédures ont tendance à se multiplier et les obligent souvent à s'interrompre : "si on a besoin d'un règlement, il faut sortir une procédure "demande de paiement", donc c'est signé, resigné, après ça part à la comptabilité, c'est re-contresigné (...) c'est quand même assez lourd » (GP B). Le fait de «tout faire signer et vérifier» participe du sentiment d'une perte d'autonomie. Outre le respect des procédures, la formalisation et le contrôle des actions réalisées par les gestionnaires ont tendance à augmenter. Au sein du service $\mathrm{C}$, les responsables d'équipe souhaitent harmoniser les pratiques : «ça va même jusqu'à la couleur des classeurs ! On doit avoir les mêmes classeurs, au même endroit sur le bureau » (GP C). Les façons de faire sont de plus en plus prescrites :

— il est demandé aux gestionnaires du service $\mathrm{C}$ de s'avancer en commençant la vérification des bulletins la veille du premier tour de paie ${ }^{5}$. Lors des observations, certaines gestionnaires vérifiaient la veille, d'autres non ;

— les responsables ont récemment demandé aux équipes de ne pas faire «tourner des paies à la demande ${ }^{6} »:$ «ils ne veulent pas qu' on le fasse, car, pour eux, on perd du temps alors que, dans bien des cas, c'est important de vérifier tout de suite» (GP C).

L'objectif de ces prescriptions est d'augmenter la productivité de chaque gestionnaire de manière à accroître le nombre de portefeuilles gérés et d'atteindre le seuil retenu par le Directeur de la performance à savoir 500 paies par personne.

Dans cette optique, les moyens de communication avec le local sont limités (via la messagerie électronique au sein du service $\mathrm{B}$ avant d'adopter l'outil Neocase déjà présent au sein du service C), les échanges sont « tracés » pour mieux identifier les «manquements » de chacune des parties. Les outils et procédures tendent également à uniformiser les façons de faire malgré la variabilité inhérente à la présence du client. Par exemple, l'outil Neocase

4 Ce regret n'est pas seulement exprimé par les gestionnaires issus du local. Des gestionnaires nouvellement recrutés évoquent également ce critère en comparaison à des expériences passées.

5 «Un tour de paie » désigne le moment où le logiciel HR Access calcule les bulletins de paie en fonction des données saisies par les gestionnaires et les correspondants.

6 «Faire tourner des paies à la demande » permet au gestionnaire de vérifier le bulletin de paie après la modification d'un élément de ce dernier sans attendre le prochain « tour de paie». 
oblige le local à «classer » ses demandes par ordre d'importance et au CSP de respecter les délais du contrat de service liés aux demandes (par exemple, les arrêts maladie doivent être traitées sous sept jours). Le local peut voir si la demande est «en attente »-dans ce cas, il manque des documents - «en cours» ou «non lue». Des feux de signalisation indiquent si le délai est respecté (vert) ou expiré (rouge). Les gestionnaires mettent progressivement en place des stratégies: «mieux vaut être dans le rouge et éviter deux manipulations par exemple si on ne connaît pas la date de reprise » (GP C) et établissent leur propre classement de priorités au-delà des indications du système. En raison de la particularité de certaines demandes, les gestionnaires ont souvent été observés en train de « reprendre la main » sur le système : "on est dans l'obligation d'intervenir, mais on le sait, dès que ce sont des cas particuliers en maladie, je suis toujours vigilante, car le système n'est pas super fiable niveau maladie... » (GP C).

De manière générale, les résultats révèlent des dispositifs organisationnels, sociaux et techniques au sein des situations $\mathrm{B}$ et $\mathrm{C}$ (en CSP) qui ont tendance à diminuer les possibilités de transformation des ressources en capabilités. Le contenu et l'organisation du travail conduisent à un confinement fort de l'activité des gestionnaires et à une perte d'autonomie. L'introduction de nouveaux outils pèse sur l'activité des gestionnaires et des correspondants et entraîne une rigidité dans les façons de faire et dans les relations. Le «client » devient une ressource de moins en moins mobilisable ainsi que les collègues, de moins en moins accessibles en raison de la spécialisation des tâches et de l'accroissement de la charge de travail. Ces différents dispositifs entravent les possibilités réelles de réaliser des fonctionnements de valeurs c'est-à-dire de faire un travail «bien fait». D'après les entretiens, à la question « que signifie, pour vous, un travail de qualité ? », les gestionnaires des CSP évoquent en priorité la satisfaction du local et du salarié (paies justes et équité de traitement). Au sein des CSP (services B et C), le sentiment de ne pas pouvoir faire un travail de qualité est exprimé lors des entretiens et observations : «on n'a plus le temps de faire des paies de qualité, c'est de la quantité, je fais du moins bon boulot qu'avant (...) Bon c'est normal, la paie on doit la sortir en temps et en heure, mais moi je veux qu'elle soit juste ma paie le plus possible»(GP B). Or cette impossibilité de faire un travail de qualité empêche la construction de sens vis-à-vis de son action professionnelle et peut être source d'atteinte à la santé (Petit \& Dugué, à paraître). Dans ce contexte, des tentatives de «reconception» dans l'usage sont observées qui vont permettre, nous allons le voir, d'accroître les possibilités de convertir les ressources en capabilités.

\section{2.- Une reconception dans l'usage}

Au fil du temps, différentes «adaptations » ont été observées visant à faire en sorte que les choses se passent au mieux compte tenu des contraintes. Elles traduisent une reconception progressive du CSP dans l'usage - reconception tolérée et parfois prise en compte.

\subsection{1.- Les enjeux de la reconception : protection d'une relation de service oubliée par la structure et atteinte d'une qualité « gérée »}

Les régulations de la première catégorie «L'organisation CSP et ses procédures » visent à atteindre une production de qualité et sont parfois également orientées vers la protection de la relation avec le client. Prenons quelques exemples.

La séparation des tâches entre les gestionnaires paie (GP) et administratif (GA) s'est rapidement révélée inefficace au sein du service $B$. L'attente et les interruptions fréquentes entre les deux types de gestionnaires étaient source de conflits fréquents. Par exemple, les GP étaient dans l'obligation d'attendre la création de la fiche d'un nouveau salarié dans le système informatique pour réaliser son bulletin de paie. Or, les gestionnaires administratifs «assistaient » plusieurs GP. Les GA recevaient alors des «pressions » de la part des GP qui souhaitaient traiter un salarié qui n'avait pas encore été créé. De plus, le système bloquait l'accès aux GP qui ne pouvaient donc pas s'avancer si la GA était occupée. Les gestionnaires paie ont exprimé leur mécontentement : « on perd un temps fou, la GA est perdue et nous on 
attend : laissez-nous les accès pour prendre la main !» (GP B). Par ailleurs, les gestionnaires nous ont dit ne plus avoir connaissance de certaines informations concernant les dossiers gérés puisque certaines informations (par exemple, une absence maladie) étaient communiquées uniquement aux GA par le local qui oubliaient parfois de les transférer aux GP : le traitement des paies se complexifiait, les délais étaient de plus en plus difficiles à tenir. Les dossiers devenaient impersonnels - chaque gestionnaire traitant un «bout» du dossier - et le traitement était considéré « opaque » par les correspondants rencontrés qui ne savaient pas toujours bien à qui envoyer les informations et «qui faisait quoi ». Les relations avec le local se dégradaient. Face aux insatisfactions largement exprimées par les gestionnaires (notamment lors des réunions de service), les responsables ont décidé de mettre progressivement fin à la séparation des tâches et de faire évoluer les GA vers des postes de GP. Les gestionnaires rencontrés après ce changement ont exprimé leur satisfaction d'avoir retrouvé une gestion de « $\mathrm{A}$ à $\mathrm{Z}$ ».

Certaines procédures sont mises en place au sein des services $\mathrm{B}$ et $\mathrm{C}$ et visent à « encadrer » les façons de faire, mais leur efficacité ou leur employabilité est parfois remise en question par le groupe d'exécution. Une gestionnaire nous expliquait avoir gardé «son » tableau pour l'envoi des acomptes et des saisies sur salaire à la comptabilité : «ce tableau convenait parfaitement à la comptable et puis un jour des gens ont dit : il faut faire une demande de règlement par saisie, une par une. J'ai refusé et aujourd'hui on revient comme avant. Il faut arrêter les trucs aux normes et se demander le temps que l'on va passer dessus » (GP B). Une autre gestionnaire partageait cet avis et considérait cette nouvelle procédure comme « une perte de temps ». Les gestionnaires nous ont rapporté que c'est lors d'une réunion d'équipe qu'une discussion a eu lieu avec les responsables et où les gestionnaires ont démontré l'inutilité et la complexité du tableau nouvellement créé. L'ancien tableau a alors été réintroduit. Les gestionnaires nous ont également rapporté avoir remis en question, lors d'une réunion d'équipe, la procédure «très lourde » de traitement des soldes de tout compte et notamment l'attente des corrections des responsables d'équipe jugées «infantilisantes ». Mais pour le moment, aucun compromis n'a été trouvé.

La seconde catégorie de régulations «La relation avec le local» vise à protéger une relation de service que la structure CSP tend à ignorer.

Tout au long de nos observations, de nombreux arrangements avaient lieu entre les gestionnaires et leur correspondant : l'interdiction de traiter des éléments au-delà du $1^{\text {er }}$ tour de paie sous le logiciel HR Access (service C) était parfois contournée et les gestionnaires saisissaient de nouveaux éléments notamment pour les centres « avec qui on s'entend bien » et pour les cas qui «risquaient d'affecter les salariés». Au sein des services B et C, les gestionnaires n'avaient pas toujours les informations nécessaires pour avancer, mais essayaient de se «débrouiller sans » de manière à «ne pas déranger » le local et produire la paie dans les délais. Par ailleurs, les observations ont révélé, malgré l'interdiction, l'usage fréquent des téléphones pour communiquer avec le local. D'après les gestionnaires, l'utilisation du téléphone permet d'obtenir «plus rapidement» les informations, mais aussi d' «éviter de laisser des traces des oublis et erreurs du correspondant», de «donner et avoir des nouvelles» ou encore de «rassurer le correspondant que les paies sont en cours de traitement » : « on n'est pas censé les avoir au téléphone, mais nous on se bat pour » (GP C). Afin de protéger la relation avec son correspondant, les gestionnaires n'hésitent pas à s'échanger les portefeuilles en fonction des affinités et des modes de fonctionnement de chacun comme l'illustre l'extrait d'un dialogue entre trois gestionnaires du service C (voir Tableau 3) - dialogue recueilli lors des observations : 
GP1 - on est le prestataire, eux les clients. S'ils adoptent le langage d'une « classe de maternelle », on ne peut pas répondre par un langage de ce type... Des demandes cordiales parfois, mais c'est rare...

GP2 - une fois on les a vus, mais c'est une réelle demande de notre part de les rencontrer !

GP3 - moi, mon correspondant, Bernard, était présenté comme pas facile, mais ça s'est passé correctement, mais quand j'ai transféré mes dossiers à GP1, il n'a pas accepté

GP1 - c'était comme si je n'existais pas ! Mais une fois que je l'ai vu, ça allait mieux. Ils sont en totale négation vis-à-vis du CSP : d'abord, ils disent non. Au départ, je n'existais pas... J'ai même reçu un mail «je ne vous connais pas, je ne travaille pas avec vous ». Là maintenant, je l'ai rencontré, ça va mieux. Ah au fait, Bernard vient à Lyon...

GP3 - ce serait sympa que l'on se voie à l'extérieur, boire un verre. GP2 tu viens ? [Rires]

GP1 et GP3 - avec GP2, ça s'est mal passé...

GP3 - non, mais c'est moi qui ait proposé ça, je trouvais ça sympa de se voir de manière informelle, d'aller prendre un verre avec lui comme la dernière fois

GP1 et GP2 - en fait on a échangé nos clients, GP2 a pris Lucie, et GP1, Bernard

GP1 - en fait moi ça m'arrange, Lucie elle demande trop de choses...

Tableau 3 : Extrait d'un dialogue entre 3 gestionnaires du service $\mathrm{C}$

Table 3: Extract from a dialogue between 3 pay administrators (service C)

Cet exemple permet de voir également que des rencontres informelles ont lieu entre les gestionnaires et leurs correspondants. Cela rejoint les souhaits largement exprimés lors des entretiens par l'ensemble des gestionnaires (services B et C, voir Tableau 4) de se rencontrer, connaître l'autre, son fonctionnement :

\begin{tabular}{|l|l|l|l|l|l|l|l|l|l|l|l|l|l|l|l|}
\hline & & $\mathrm{P} 1$ & $\mathrm{P} 2$ & $\mathrm{P} 3$ & $\mathrm{P} 4$ & $\mathrm{MP} 1$ & $\mathrm{MP2}$ & $\mathrm{P} 5$ & $\mathrm{P} 6$ & $\mathrm{MP3}$ & $\mathrm{P} 7$ & $\mathrm{P} 8$ & $\mathrm{P} 9$ & $\mathrm{P} 10$ & TOTAL \\
\hline $\begin{array}{l}\mathrm{Si} \text { vous deviez } \\
\text { proposer des pistes connaître, se voir } \\
\text { d'amélioration? }\end{array}$ & $\begin{array}{l}\text { Importance } \\
\text { téléphone }\end{array}$ & 0 & & & & & 0 & & & 0 & 0 & 0 & 0 & 7 \\
\hline
\end{tabular}

Tableau 4 : Sur les 13 opérateurs interrogés (service B et C), 7 évoquent le souhait de se rencontrer et de se connaître, 5 énoncent l'importance du téléphone

Table 4: Out of 13 operators interviewed (pay services $B$ and $C$ ), 7 evoke the desire to meet each other and 5 underlined the importance of the phone

Aussi les opérateurs mettent-ils progressivement en place des facteurs de conversion afin de pouvoir convertir l' «organisation du travail» et le «client» en possibilités concrètes de faire un travail de qualité. En effet, l'objectif des diverses régulations mises en évidence est avant tout de parvenir à faire un travail qui remplit les critères de qualité précédemment énoncés : la satisfaction du client et du salarié. Pour les gestionnaires, le client est avant tout un partenaire de l'activité, une ressource. Différentes tentatives sont alors observables (appels téléphoniques, réunions informelles...) qui visent la transformation de cette ressource en capabilités permettant la réalisation effective d'une paie de qualité, dans les délais. Aussi les gestionnaires ne vont-ils pas hésiter à appeler leur correspondant si une information urgente manque au dossier d'un salarié, même si les délais prévus par le contrat ou le système sont dépassés. Nous avons également pu observer une gestionnaire qui appelait directement les salariés : «normalement on n'a pas le droit, mais c'est pour aider la personne, le pauvre, il était perdu [cas d'un départ en retraite où le salarié ne parvenait pas à établir son dossier]. C'est pour bien faire, pas pour nuire à la société ! »(GP B).

\subsection{2.- Vers une modification de la structure « CSP »?}

Nous venons de mettre en évidence différentes tentatives positives d'amélioration. Nous allons maintenant analyser de quelle manière ces tentatives (niveau 3) ont - ou non modifié les niveaux 2 (les choix de l'établissement local) et 1 (la structure, la stratégie) (Petit et al., 2011). 
Nos observations révèlent que des compromis s'élaborent entre le niveau 3 (les opérateurs au quotidien) et le niveau 2 (l'établissement local et son organisation) : les responsables mettent fin à la séparation des tâches (service B) ou ne l'appliquent pas (service C), des réunions de service permettent de renégocier des procédures et de changer les façons de faire initialement décidées (service B). D'autres adaptations sont tolérées au quotidien : les appels ou les échanges de portefeuille se font avec l'accord tacite du management. Mais les adaptations relevant de la catégorie «La relation avec le local» apparaissent toutefois davantage «clandestines»: les contournements du logiciel, les rencontres officieuses ne sont pas - ou peu - discutés. Autrement dit, la relation client-fournisseur qui relève du niveau 1 (la structure, la stratégie) n'est pas véritablement mise en discussion malgré les difficultés qu'elle engendre au quotidien. Dans ce cadre, les régulations sont coûteuses pour les salariés qui «luttent» quotidiennement contre les règles et procédures pour réaliser un travail de qualité «pour soi » (paies justes, dans les délais, traitement équitable) et «pour le client » (satisfaction facilitant les coopérations et les compromis).

Le tableau ci-dessous propose un récapitulatif de l'ensemble des régulations opérées par les gestionnaires paie (services B et C) et les objectifs sous-jacents. Il précise également si ces régulations font l'objet de débats au sein des services :

\begin{tabular}{|c|c|c|c|c|}
\hline $\begin{array}{l}\text { Catégories } \\
\text { de } \\
\text { régulations }\end{array}$ & $\begin{array}{l}\text { Sous- } \\
\text { catégories }\end{array}$ & Exemples et verbatims & Objectifs & $\begin{array}{l}\text { Objets de } \\
\text { débats? }\end{array}$ \\
\hline \multirow[t]{2}{*}{$\begin{array}{l}\text { L'organisati } \\
\text { on CSP et } \\
\text { ses } \\
\text { procédures }\end{array}$} & $\begin{array}{l}\text { Remise en } \\
\text { question de } \\
\text { l'organisation } \\
\text { du travail }\end{array}$ & $\begin{array}{l}\text { - Remise en question de la séparation GA/GP } \\
\text { " on perd un temps fou, la GA est perdu et nous, on attend, laissez-nous } \\
\text { les accès pour prendre la main !" }\end{array}$ & $\begin{array}{l}\text { Fin attente travail de l'autre } \\
\text { Gestion globale } \\
\text { Plus d'autonomie } \\
\text { Respect délais de traitement }\end{array}$ & Oui \\
\hline & $\begin{array}{l}\text { Non } \\
\text { application de } \\
\text { procédures }\end{array}$ & $\begin{array}{l}\text { Non application de tableaux, formulaires, prescriptions de façons } \\
\text { de faire... } \\
\text { "nous, dans l'équipe on ne voit pas l'utilité de faire ça, on ne le fait } \\
\text { pas" } \\
\text { "en } 2009 \text {, il fallait fournir un état d'avance des ses payes mais ça n'a } \\
\text { pas duré longtemps. Tout le monde a eu le sentiment de se faire tracer»" }\end{array}$ & $\begin{array}{l}\text { Plus d'autonomie } \\
\text { Choix façons de faire } \\
\text { Gain de temps }\end{array}$ & $\begin{array}{l}\text { Oui dans } \\
\text { certains cas }\end{array}$ \\
\hline \multirow[t]{3}{*}{$\begin{array}{l}\text { Relation } \\
\text { CSP-Local }\end{array}$} & $\begin{array}{l}\text { Non respect } \\
\text { des consignes } \\
\text { de communi- } \\
\text { cation }\end{array}$ & $\begin{array}{l}\text { - Utilisation fréquente du téléphone pour appeler le correspondant } \\
\text { - Utilisation occasionnelle du téléphone pour appeler le salarié } \\
\text { - Organisation de rencontres informelles } \\
\text { " on n'est pas censé les avoir au téléphone mais nous, on se bat pour " } \\
\\
\text { " c'est moi qui ait proposé ça, je trouvais ça sympa de se voir de manière } \\
\text { informelle, d'aller prendre un verre avec lui comme la dernière fois " }\end{array}$ & $\begin{array}{l}\text { Rapidité } \\
\text { Relationnel } \\
\text { Satisfaction salarié } \\
\text { Se voir / Se comprendre }\end{array}$ & Non \\
\hline & $\begin{array}{l}\text { Arrangements } \\
\text { avec les } \\
\text { correspondants }\end{array}$ & $\begin{array}{l}\text { - Traitement éléments de paye au-delà des délais } \\
\text { - Traitement des demandes non formulées sous Neocase } \\
\text { « je suis très arrangeante avec l'exploitant » } \\
\text { « il faut qu'il passe par Neocase ... bon on a des seuils de tolérance » }\end{array}$ & $\begin{array}{l}\text { Relationnel } \\
\text { Satisfaction salariés } \\
\text { Gain de temps }\end{array}$ & Non \\
\hline & $\begin{array}{l}\text { Nouvelle } \\
\text { répartition des } \\
\text { portefeuilles } \\
\text { en interne }\end{array}$ & $\begin{array}{l}\text { Répartition des portefeuilles en fonction des affinités } \\
\text { "en fait, on a échangé nos clients, GP2 a pris Lucie et GPA, Bernard " }\end{array}$ & Relationnel & Non \\
\hline
\end{tabular}

Tableau 5 : Récapitulatif des régulations effectuées par les gestionnaires au sein des CSP

Table 5: Summary of regulations laid down by the pay administrators in SSC

\section{6.- Discussion et proposition d'intervention}

\section{1.- Le changement et les tentatives de reconception organisationnelle}

Le changement ici étudié s'est imposé comme une évidence dans la stratégie globale de réduction des coûts de fonctionnement dans laquelle le groupe s'était engagé. La nouvelle organisation visée existait déjà, il s'agissait même d'un «modèle » qu'il convenait d'appliquer puisqu' «il a fait ses preuves ». Cette application s'est traduite par l'élaboration d'un contrat de service. Le contrat signé entre le CSP et le local a pris la forme particulière d'une relation client-fournisseur où il n'y a pas d'objectif d'apprentissage puisque les connaissances et compétences sont supposées suffisantes de part et d'autre (Sardas, 2002). 
Le besoin du client étant censé ne pas évoluer, l'activité de service tend alors vers la rationalisation de l'offre puisque le problème est censé être bien défini (Barcet \& Bonamy, 1995). Dans ce contexte, la nouvelle organisation cherche à s'industrialiser: "l'idée d'industrialisation désigne un processus au cours duquel une catégorie d'organisation n'appartenant pas au monde industriel tend à se rapprocher de ce dernier » (Gadrey, 1994, pp. 167-168). Les thèmes organisationnels associés à l'organisation mécaniste - modèle de référence au sein de l'industrie - apparaissent très proches de la structure CSP (situation B et C) : le travail opérationnel est réglé selon des procédés de travail standardisés et spécialisés, la division du travail est très poussée, la production est une production de masse rendue possible par la mutualisation des fonctions support de l'ensemble des divisions, la ligne hiérarchique est développée, les méthodes de gestion et de mesure des performances sont liées à la recherche prioritaire de gains de productivité et l'environnement est stabilisé - en apparence - par l'existence du contrat. L'interaction entre les co-contractants est elle-même «machinisée »: le courrier électronique ou l'outil Neocase se substituent aux relations physiques et téléphoniques directes susceptibles de faire perdre du temps. La production de la paie est censée pouvoir se réaliser sans relation directe entre les co-contractants, sans «relation de service». La seule solution technique apparaît suffisante pour réaliser la prestation (Hubault \& Bourgeois, 2002). Cette volonté de se rapprocher des modèles industriels peut «tenir de la fuite devant le risque d'un abandon d'archétypes éprouvés, au manque de connaissances des réalités opérationnelles » (Lorino, 2005, p. 245).

Ce sont précisément les « réalités opérationnelles » qui ont montré rapidement les limites de cette conception du service tant les relations entre les deux unités apparaissent nombreuses et garantes d'un meilleur fonctionnement. L'analyse de l'existant (la situation A avant le passage en CSP) montre à quel point la qualité de la relation, rendue possible par des espaces d'autonomie et de liberté (interlocuteurs variés, moyens de communication illimités, etc.), est essentielle pour réaliser un travail de qualité. Au sein des CSP, les ressources et facteurs de conversion tendent à diminuer. Pourtant, les besoins et demandes du client restent instables et les gestionnaires souhaitent reconstituer du lien, comprendre le sens de leurs actions. De toute évidence, clients et fournisseurs doivent coopérer pour produire une paie de qualité et satisfaire les salariés (traitement équitable, virement des salaires en temps et en heure, bulletins lisibles, etc.). Pour coopérer, les gestionnaires tentent, au mieux, de protéger une relation de service «empêchée » par les préceptes de la structure CSP et le contrat de service. En interne, ces tentatives se concrétisent par la quête d'autonomie et la maîtrise des «portefeuilles» de « $\mathrm{A}$ à $\mathrm{Z}$ ». Vis-à-vis du client, ces tentatives se traduisent par des contournements, des appels, des rencontres informelles... Si les premières tendent à trouver un écho et modifient progressivement les préceptes de la structure initiale, les secondes sont plus «clandestines"; de ce fait, la visibilité sur le travail et les besoins des «collèguesclients » reste faible du fait d'une distance physique et organisationnelle forte.

\section{2.- Soutenir la reconception organisationnelle dans l'usage : une mise en route positive et durable des facteurs de conversion}

À ce stade de l'étude, l'enjeu est de mettre en place une intervention ergonomique visant à soutenir la reconception dans l'usage observé afin de replacer les acteurs au centre du changement. Des restitutions auprès des équipes de management et de direction ont tout d'abord permis de mettre en évidence ces tentatives positives d'amélioration et leurs rôles. Ces restitutions ont ensuite engendré la discussion et la construction communes d'une intervention ergonomique visant la mise en place d'un dispositif de «mise en route » de facteurs de conversion positifs et durables. Si les gestionnaires ont progressivement modifié l'organisation du travail et certaines procédures en interne facilitant le travail au quotidien, la compréhension et la visibilité du travail de l'autre restent, en revanche, insatisfaisantes aussi bien pour les gestionnaires que pour leurs correspondants. Dans ce contexte une méthodologie particulière, appelée la «co-analyse constructive des pratiques », a été mise au 
point. À travers cette pratique réflexive, chacun des acteurs est amené à observer l'activité de son partenaire à un moment clef du processus paie décidé conjointement : le jour de l'envoi des prépaies en local et le lendemain au CSP. Il est demandé à l'opérateur visité de verbaliser son activité : un travail d'extériorisation est attendu où l'activité est réifiée, rendue extérieure à l'opérateur (Falzon, 2005). La verbalisation est ici adressée au partenaire. Ce dernier est invité à poser toutes les questions qu'ils souhaitent à partir d'une incompréhension et/ou d'un effet de surprise. La pratique réflexive s'apparente ainsi à une allo-confrontation croisée (Mollo \& Falzon, 2004) qui repose ici sur l'activité de l'autre en situation. Il a été décidé que l'ergonome serait en charge de l'organisation des binômes et serait présent, un temps, pour recueillir les échanges. Les résultats alimenteraient un groupe de travail naissant au sein de la division Transport intitulé : "Comment travailler ensemble avec le CSP ? Comment mieux s'articuler? » auquel l'ergonome serait associé.

Plus généralement, l'enjeu est de reconcevoir progressivement une organisation « instrumentalisable » c'est-à-dire une organisation qui se prête à l'adaptation d'elle-même. Pour cela le rôle de l'ergonome est double. Dans un premier temps, les ressources existantes et les facteurs de conversion organisationnels et individuels en présence (qui entravent ou, au contraire, favorisent l'usage effectif de ces ressources) sont mis à jour. Dans un second temps, un dispositif de mise en route de facteurs de conversion positifs est à construire en fonction de la situation et des besoins. Ainsi, il s'agit non de «conduire » ou «piloter » un changement, mais de l'«accompagner » voire de le «catalyser» (Lorino, 2005 ; Petit, 2005) en provoquant un processus d'apprentissage et de développement. L'intervention consiste ici à « catalyser » le changement en enclenchant un processus de conversion des ressources en capabilités. La «cible» de l'intervention devient alors les «facteurs de conversion » qui vont permettre aux personnes de se développer et de réaliser des fonctionnements de valeur synonymes d'un travail de qualité. L'analyse ergonomique facilite la mise en évidence de ces facteurs et du coût généré par le travail et les régulations lorsque ces facteurs sont négatifs. Le fait de rétablir et développer les facteurs de conversion pourrait contribuer à concevoir et anticiper une organisation « capacitante », source de développement, de réussite et de progrès. Cette perspective s'inscrit dans le courant de l'ergonomie constructive (Falzon, 2013) où l'intervention a comme moyen et finalité le développement conjoint des individus et des organisations (Petit \& Coutarel, 2013; Petit \& Dugué, à paraître).

\section{BIBLIOGRAPHIE}

Autissier, D., \& Wacheux, F. (2000). Structuration et management des organisations : Gestion de l'action et du changement dans les entreprises. Paris: L'Harmattan.

Barcet, A., \& Bonamy, J. (1995). Chronique des services - Quel(s) service(s) pour quelle société ? Revue d'Economie Industrielle, 72(2), 111-117.

Béguin, P. (2008). Conception et santé : Quelques remarques sur le statut de l'activité de travail dans la conception des systèmes de production. Psychologie du Travail et des Organisations, 14(4), 369-384.

Béguin, P. (2013). La conception des instruments comme processus dialogique d'apprentissages mutuels. In P. Falzon (Ed.), Ergonomie constructive (pp. 147-160). Paris: PUF.

Béguin, P., \& Cerf, M. (2004). Formes et enjeux de l'analyse de l'activité pour la conception des systèmes de travail. Activités, 1(1), 54-71. http://activites.org/v1n1/beguin.pdf

Bernoux, P. (2004). Sociologie du changement dans les entreprises et les organisations. Paris : Seuil.

Bonvin, J. M., \& Farvaque, N. (2007). L'accès à l'emploi au prisme des capabilités, enjeux théoriques et méthodologiques. Formation Emploi, 98, 9-23.

Boussard, V. (2013). Le rôle des consultants dans la diffusion des transformations organisationnelles. In J. Petit, K. Chassaing, \& S. Aubert (Eds.), Des pratiques en évolution (pp. 440-447). Toulouse: Éditions Octarès.

Cerf, M., \& Falzon, P. (2005). Situations de service : Travailler dans l'interaction. Paris: PUF.

Coutarel, F. (2004). La prévention des troubles musculo-squelettiques en conception : Quelles marges 
de mancuvre pour le déploiement de l'activité ? Thèse de doctorat d'Ergonomie, Bordeaux: Université Victor Segalen Bordeaux 2.

Coutarel, F., \& Petit, J. (2009). Le réseau social dans l'intervention ergonomique : Enjeux pour la conception organisationnelle. Management et Avenir, 7(27), 135-151.

Cuvelier, L. (2007). Changement organisationnel et technique : Étude de l'appropriation d'une stratégie opératoire par un collectif de travail. Mémoire de Master 2 Recherche d'Ergonomie, Paris: CNAM.

Daniellou, F. (1992). Le statut de la pratique et des connaissances en ergonomie. Habilitation à Diriger des Recherches, Bordeaux: Université Victor Segalen Bordeaux 2.

Daniellou, F. (2006). Les mondes du travail. In L. Théry (Ed.), Le travail intenable (pp. 19-80). Paris: La Découverte.

Detchessahar, M., Clergeau, C., Devigne, M., Dumond, J. P., Honoré, L., \& Journe, B. (2006). Transformation des organisations et santé des salariés : Proposition d'un programme de recherche. $17^{\text {ème }}$ Congrès de l'AGRH, Reims, France, novembre.

DiMaggio, P. J., \& Powell, W. W. (1983). The iron cage revisited: Institutional isomorphism and collective rationality in organizational fields. American Sociological Review, 48, 147-160.

Dugué, B. (2006). La folie du changement. In L. Théry (Ed.), Le travail intenable (pp. 95-118). Paris: La Découverte.

Falzon, P. (1994). Les activités méta-fonctionnelles et leur assistance. Le Travail Humain, 57(1), 123.

Falzon, P. (1996). Des objectifs de l'ergonomie. In F. Daniellou (Ed.), L'ergonomie en quête de ses principes : Débats épistémologiques (pp. 233-242). Toulouse: Éditions Octarès.

Falzon, P. (2005). Ergonomie, conception et développement. Conférence introductive. 40ème Congrès de la SELF, Saint-Denis, La Réunion, septembre.

Falzon, P. (2013). Ergonomie constructive. Paris: PUF.

Falzon, P., Nascimento, A. \& Pavageau, P. (2008). Towards models and tools for assessing the developmental quality of work. In L. Sznelwar, F. Mascia, \& U. Montedo (Eds.), Human Factors in Organizational Design and Management - IX. Sao Paulo, Brésil, mars.

Falzon, P., Sauvagnac, C., \& Chatigny, C. (1996). Collective knowledge elaboration. COOP'96, Juanles-Pins, France, juin.

Fernagu-Oudet, S. (2012). Favoriser un environnement « capacitant» dans les organisations. In E. Bourgeois, \& M. Durand (Eds.), Former pour le travail (pp. 201-213). Paris: PUF.

Gadrey, J. (1994). La modernisation des services professionnels. Rationalisation industrielle ou rationalisation professionnelle ? Revue Française de Sociologie, 35(2), 163-195.

Giddens, A. (1987). La constitution de la société : Éléments de la théorie de la structuration. Paris: PUF.

Gilbert, P. (2008). Apprendre dans les réorganisations : Une perspective communicationnelle. Communication et Organisation, 33, http://communicationorganisation.revues.org/435

Guibert, S. (2009). Évaluation ergonomique de la performance d'un changement organisationnel. Thèse de doctorat d'Ergonomie, Paris: CNAM.

Hubault, F., \& Bourgeois, F. (2002). La relation de service : Une convocation nouvelle pour l'ergonomie? In. F. Hubault (Ed.), La relation de service, opportunités et questions nouvelles pour l'ergonomie (pp. 5-31). Toulouse: Octarès.

Leplat, J. (2004). Éléments pour l'étude des documents prescripteurs. Activités, 1(2), 195-216. http://www .activites.org/v1n2/Leplat.pdf

Lorino, P. (2005). Piloter ou catalyser le changement organisationnel : Une approche sémiotique et pragmatique. In J. C. Sardas, \& A. M. Guénette (Eds.), Sait-on piloter le changement ? (pp. 221253). Paris: L'Harmattan.

Lorino, P. (2009). Concevoir l'activité collective conjointe : L'enquête dialogique. Étude de cas sur la sécurité dans l'industrie du bâtiment. Activités, 6(1), 87-110, http://activites.org/v6n1/lorino.pdf

Mollo, V., \& Falzon, P. (2004). Auto- and allo-confrontation as tools for reflective activities. Applied Ergonomics, 35(6), 531-540. 
Pavageau, P., Nascimento, A., \& Falzon, P. (2007). Les risques d'exclusion dans un contexte de transformation organisationnelle. PISTES, 9(2). http://www.pistes.uqam.ca/v9n2/pdf/v9n2a6.pdf

Petit, J. (2005). Organiser la continuité du service : Intervention sur l'organisation d'une mutuelle de santé. Thèse de doctorat d'Ergonomie, Bordeaux : Université Victor Segalen Bordeaux 2.

Petit, J., \& Coutarel, F. (2013). L'intervention comme dynamique de développement conjoint des acteurs et de l'organisation. In P. Falzon (Ed.), Ergonomie constructive (pp. 133-146). Paris: PUF.

Petit, J., \& Dugué, B., (à paraître). Quand l'organisation empêche un travail de qualité : Étude de cas. PISTES.

Petit, J., Dugué, B., \& Daniellou, F. (2011). L’intervention ergonomique sur les risques psychosociaux dans les organisations : Enjeux théoriques et méthodologiques. Le Travail Humain, 4(74), 391-409.

Rabardel, P. (1995). Les hommes et les technologies, approche cognitive des instruments contemporains. Paris: Armand Colin.

Rasmussen, J. (1997). Risk management in a dynamic society: A modeling problem. Safety Science, 27(2-3), 183-213

Reynaud, J. D. (1988). Les régulations dans les organisations : Régulation de contrôle et régulation autonome. Revue française de sociologie, XXIX, 5-18.

Reynaud, J. D. (1995). Le conflit, la négociation et la règle. Toulouse: Éditions Octarès.

Sardas, J. C. (2002). Relation de partenariat et recomposition des métiers. In F. Hubault (Ed.), La relation de service, opportunités et questions nouvelles pour l'ergonomie (pp. 209-224). Toulouse: Octarès.

Sardas, J. C., \& Guénette, A. M. (2005). Avant-Propos. In J. C. Sardas, \& A. M. Guénette (Eds.), Saiton piloter le changement? (pp. 11-26). Paris: L'Harmattan.

Sardas, J. C., \& Lefebvre, P. (2005). Théories des organisations et interventions dans les processus de changement. In J. C. Sardas, \& A. M. Guénette (Eds.), Sait-on piloter le changement? (pp. 255 289). Paris: L'Harmattan.

Schulz, V., Hochstein, A., Uebernickel, F., \& Brenner, W. (2009). Definition and classification of ITShared-Service-Center. 15th Conference on Information Systems. San-Francisco, California, August.

Sen, A. (2009). The idea of justice. London: Penguin Books Ltd.

Terssac, G. (de) (2003). Travail d'organisation et travail de régulation. In G. de Terssac (Ed.), La théorie de la régulation sociale de Jean-Daniel Reynaud. Débats et prolongements (pp. 121-134). Paris: La découverte.

Terssac, G. (de), \& Lompré, N. (1996). Pratiques organisationnelles dans les ensembles productifs : Essai d'interprétation. In J.C. Sperandio (Ed.), L'ergonomie face aux changements technologiques et organisationnels du travail humain (pp. 51-70). Toulouse: Éditions Octarès.

Valot, C. (2001). Pour une ergonomie du changement dans les organisations. Comptes rendus du Congrès SELF ACE 2001, Les transformations du travail, enjeux pour l'ergonomie, 3, 24-29.

Villarmois (de La), O., \& Tondeur, H. (2002). Externalisation et centre de services partagés : Deux formes d'organisation de la fonction comptable sur un même continuum. Revue Française de Comptabilité, 348, 35-38.

Wilson, J. R. (2012). Fundamentals of systems ergonomics. Work, 41, 3861-3868.

\section{RESUME}

Cet article cherche à décrire les processus de reconception organisationnelle dans l'usage réalisés par les opérateurs à la suite d'un changement organisationnel. Ces processus visent à assurer une production de qualité et à protéger des coopérations que la structure, telle qu'elle a été conçue, a eu tendance à ignorer. La méthodologie qualitative mise en œuvre identifie les caractéristiques de la structure et met en évidence le rôle des processus de reconception identifiés. Les principaux résultats montrent que les opérateurs tentent de convertir les ressources de la nouvelle organisation en «capabilités » c'est-à-dire en possibilités effectives « de faire » et « de faire bien ». Pour autant, la rigidité de 
la structure gêne ces tentatives qui ne sont ni toujours connues, ni discutées. L'enjeu de l'intervention ergonomique est de mettre à jour et en discussion ces tentatives afin de mettre en place une méthodologie ayant pour but de soutenir la reconception organisationnelle dans l'usage et de replacer les acteurs au cœur du changement.

\section{MOTS-CLES}

Organisation, changement organisationnel, reconception dans l'usage, capabilités, intervention ergonomique

\section{REFERENCEMENT}

Arnoud, J., \& Falzon, P. (2013). Changement organisationnel et reconception de l'organisation: des ressources aux capabilités. Activités, 10(2), 109-130, http://www .activites.org/v10n2/v10n2.pdf

Article soumis le 23 mars 2013, accepté pour publication le 28 août 2013. 